\title{
Optical vs. direct sorption and swelling measurements for the study of stiff-chain polymer-penetrant interactions
}

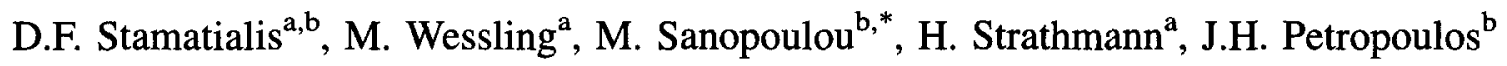 \\ ${ }^{a}$ Department of Chemical Technology, University of Twente, 7500 Enschede, Netherlands \\ bInstitute of Physical Chemistry, Demokritos National Research Centre, 15310 Aghia Paraskevi Athens, Greece
}

Received 12 September 1996; received in revised form 12 December 1996; accepted 13 December 1996

\begin{abstract}
This work involves interferometric 'optical thickness' and refractive index measurements performed in an optical thickness meter (OTM), on supported cellulose acetate (CA) films equilibrated with various activities of methylene chloride (MC) vapor. The relevant equilibrium sorption and volume swelling isotherms were determined by application of the Claussius-Mossotti equation on the assumption that these films swell unidimensionally along the thickness direction, and were compared with corresponding direct equilibrium sorption (weight gain), elongation and thickness dilation measurements on similar free films performed in a vacuum sorption/swelling apparatus (VSA) and complemented with refractive index data. Combined elongation and thickness dilation data from the VSA showed that free glass-cast CA films exhibit pronounced swelling anisotropy. The said anisotropy, although it cannot be completely eliminated, by conditioning at high degrees of swelling, does not appear to affect the extent of volume swelling significantly, thus permitting quantitative comparison of sorption and swelling isotherms determined by the VSA and the OTM. Such comparison showed satisfactory agreement between these two sets of results up to an MC uptake of ca. $0.4 \mathrm{gMC} / \mathrm{cm}^{3}$ of dry CA corresponding to a degree of swelling of ca. 0.2. Increasing discrepancies are observed at higher MC concentrations, which are attributable to breakdown of the assumption used that the supported films swell unidimensionally along the thickness direction. The present CA-MC volume swelling data exhibit the negative deviation from volume additivity on mixing typical glassy polymers.
\end{abstract}

Keywords: Solubility; Interferometry; Longitudinal and thickness swelling

\section{Introduction}

Sorption of micromolecular species by glassy polymers occurs simultaneously by dissolution in the polymer matrix, as observed above $T_{\mathrm{g}}$, and by filling the excess free volume which characterizes glassy polymers [1-5]. The latter process causes in volume

*Corresponding author. Tel: +30-1-6513111-515; fax: +30-16511766; e-mail: sanopoul@cyclades.nrcps.ariadne-t.gr. dilation of the polymer to be substantially less than expected on the basis of volume additivity of the polymer-liquid penetrant mixture [3-7], as well as marked deviation from linearity of the corresponding refractive index-composition relation [6-9].

Accordingly, useful information for the fundamental understanding and successful modeling of the mechanism of sorption can be derived if equilibrium sorption measurements are supplemented with corresponding polymer volume swelling and/or refractive 
index data. The utility of most of the data of this type which have been accumulated so far is dependent on some assumption, the general validity of which cannot be taken for granted a priori. In particular:

(a) Because of the possibility of substantial swelling anisotropy of stiff-chain polymer films (due to the tendency for preferred in-plane macromolecular orientation, to a degree dependent on film properties and method of preparation), reliable evaluation of the volume swelling of free (unconstrained) films requires parallel elongation and thickness dilation measurements. The latter are much more demanding in terms of experimental technique and are still a rarity [10]. It is much more common to rely on elongation measurements alone, assuming that any swelling anisotropy is eliminated upon annealing of the film by either heat treatment above $T_{\mathrm{g}}[4,11]$ or by plasticization with a suitable swelling agent (a suitably high concentration of the penetrant under study being very often used for this purpose) $[5,12,13]$. Our experience indicates that such a (rather vaguely formulated) assumption cannot be relied upon without a careful check in each particular case. For example, the swelling anisotropy of glass-cast cellulose acetate membranes was found to be markedly reduced by swelling in methanol - but the residual anisotropy was still substantially more pronounced than that of mercury-cast membranes [14]. The latter, though much more nearly isotropic, still exhibited significant preferred in-plane molecular orientation (revealed by measuring the birefringence of a cross section of the film [15]) - which was reduced but not completely eliminated after repeated protracted strong swelling by acetone vapor [16]. Note, incidentally, that a proposed use of elongation measurements as an experimentally simpler substitute for gravimetric sorption data is subject to uncertainties of this type [17].

(b) Volume swelling of 'supported' films (i.e. films left adhering to the casting plate after solvent evaporation is complete) is evaluated on the assumption of unidimensional dilation along the thickness direction. Although this assumption appears to hold good for very thin, weakly swollen films $[6,7,18]$, it requires checking in the case of strongly swollen and/or thicker films.

(c) On the other hand, the suppression of longitudinal swelling in supported films leaves open the question whether such films may always be assumed to attain full volume swelling equilibrium at a given activity.

In the present paper, the points (a)-(c) raised above are investigated in detail for a typical glassy polymer strong swelling agent system. The work described involves interferometric 'optical thickness' and refractive index measurements on cellulose acetate (CA) films, prepared by casting on a glass plate and left adhering to the casting plate after evaporation of the solvent. These supported films were equilibrated with various activities of methylene chloride (MC) vapor and the resulting inferred equilibrium sorption and swelling isotherms were compared with corresponding direct equilibrium sorption (weight gain), elongation and thickness dilation measurements on similar free films (i.e. films detached from the casting plate at a suitable stage of the solvent evaporation step).

\section{Experimental}

\subsection{Materials}

Cellulose acetate (CA) powder of $39.8 \%$ acetyl content (ca. 2.45 acetyl groups per glucose monomer unit) was supplied by Eastman Chemicals (code name E398-30) with the following specifications: melting range $230-260^{\circ} \mathrm{C} ; T_{\mathrm{g}}=189^{\circ} \mathrm{C}$; viscosity (measured according to ASTM D-871 (Formula A) and D1343) 114 poise; density, $\rho_{\mathrm{P}}=1.31 \mathrm{~g} / \mathrm{cm}^{3}$ and refractive index, $n_{\mathrm{P}}=1.4700$ at $25^{\circ} \mathrm{C}$.

All the films were prepared by casting a $20 \%$ solution of the CA powder in acetone (of analytical grade) which was spread with a knife blade on a clean glass surface. After evaporation of most of the solvent, the films used for the weight gain and dilation measurements were taken off the glass plate and solvent evaporation was completed in the open atmosphere and then in vacuum for several days, until the weight of the films became constant. For the interferometric measurements, the membrane remained on the casting plate and the solvent was allowed to evaporate initially in a slow $\mathrm{N}_{2}$ stream and finally in a vacuum oven at $70^{\circ} \mathrm{C}$ for several days, until the measured optical film thickness became constant.

Methylene chloride (MC) of analytical grade was used (density, $\rho_{\mathrm{c}}=1.325 \mathrm{~g} / \mathrm{cm}^{3}$ and refractive index, $n_{\mathrm{c}}=1.4200$ at $25^{\circ} \mathrm{C}$ ). 


\subsection{Methods}

2.2.1. Optical thickness and refractive index measurements on supported films

The 'optical thickness meter' (OTM) of Carl Zeiss, based on a design by Fizeau [19] was employed. This instrument uses interference of white light in reflection with incidence at an angle $\varphi$. Transmission of light from the source (halogen lamp, Philips 7027, range $400-1018 \mathrm{~nm}$ ) to the specimen, and of the resulting interference signal from the specimen to the detector is affected by suitable fiber optics. The detector consists of a multichannel spectrometer (MCS 400), which is a polychromator with concave holographic grating and a diode array (512 diodes at a center-to-center spacing of $0.8 \mathrm{~nm}$ ). A bus interface is used to connect it with a computer, where the signal is subjected to fast Fourier transformation for the calculation of the optical thickness $L$ given by

$L=2 l \sqrt{n^{2}-\sin ^{2} \varphi}$
In Eq. (1), $n$ is the refractive index, and $l$ the geometrical thickness of the specimen.

The desired MC vapor activity $a_{i}$ (taken equal to the relative vapor pressure) was generated by mixing nitrogen with saturated MC vapor in appropriate proportions at $25^{\circ} \mathrm{C}$ and passing this mixture at a rate of $1 \mathrm{l} / \mathrm{min}$ (controlled by a suitable mass flow controller), through a box thermostatted at $25^{\circ} \mathrm{C}$. The latter contained the polymer membrane adhering to the glass casting plate, with its upper surface exposed to the gas stream (see Fig. 1).

Thanks to a special design which made it possible to measure the optical thickness of the membrane using two different angles of incidence, namely $\varphi=0^{\circ}$ and $\varphi=45^{\circ}$. Eq. (1) yields

$L\left(\varphi=0^{\circ}\right)=L_{\mathrm{a}}=2 \ln$

$L\left(\varphi=45^{\circ}\right)=L_{\mathrm{b}}=2 l \sqrt{n^{2}-0.5}$

Using Eqs. (2) and (3), the refractive index and the thickness of the membrane were determined in the dry

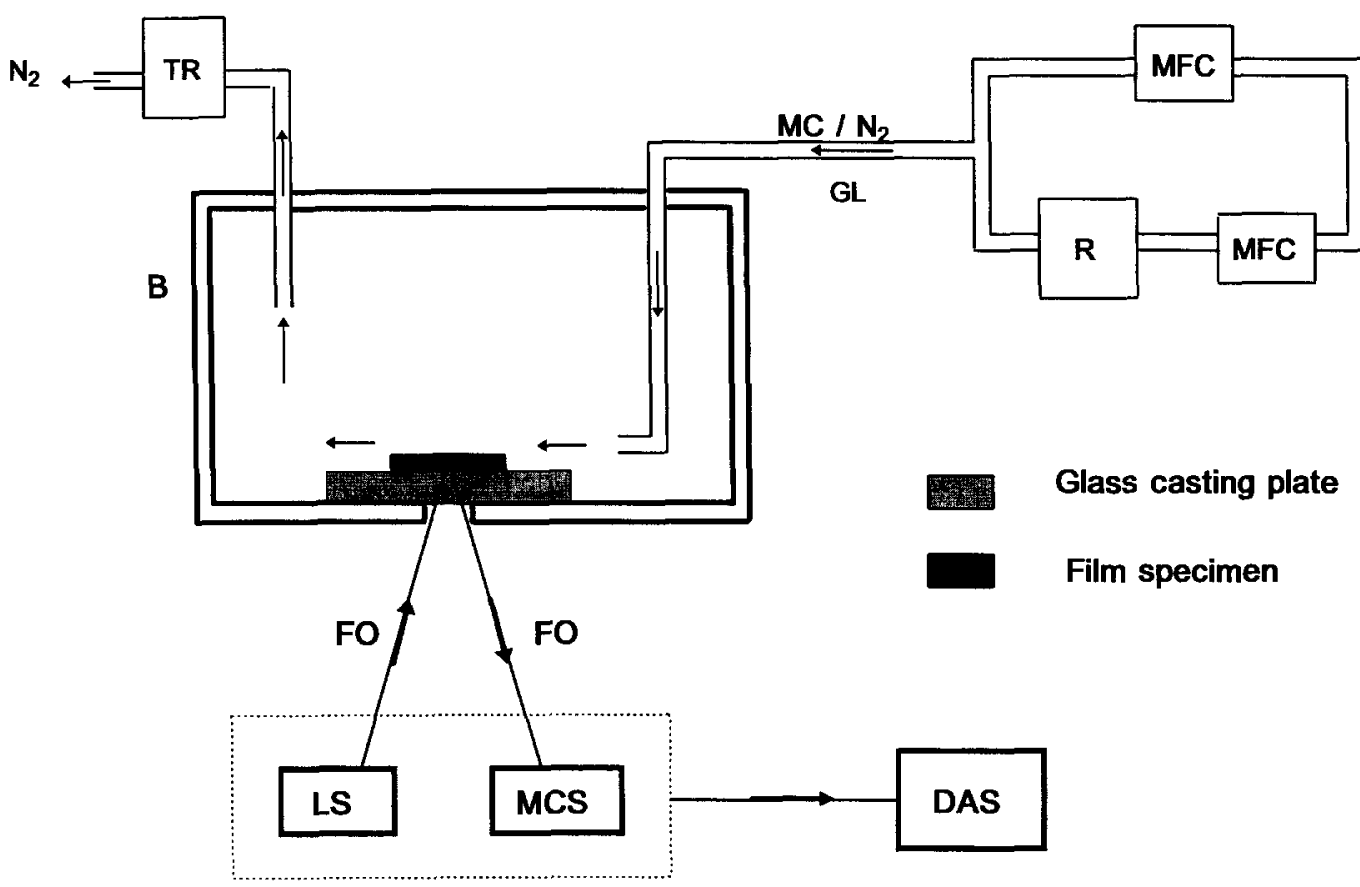

Fig. 1. Schematic representation of the apparatus for the optical thickness and refractive index measurements on supported films (OTM). LS: light source; FO: fiber optics; MCS: multi channel spectrometer; DAS: data aquisition system; MFC: mass flow controller; R: thermostatted MC reservoir; GL: thermostatted gas line; B: thermostatted box; and TR: MC trap. 


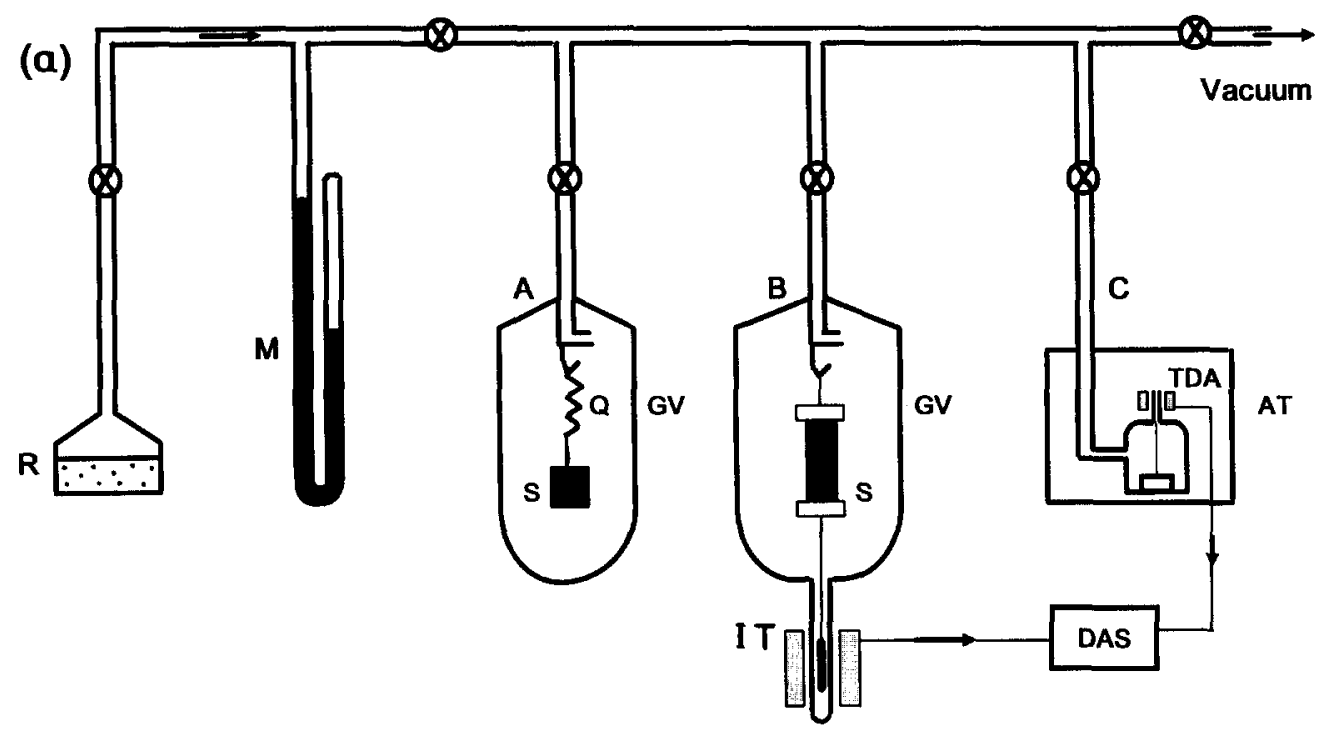

(b)
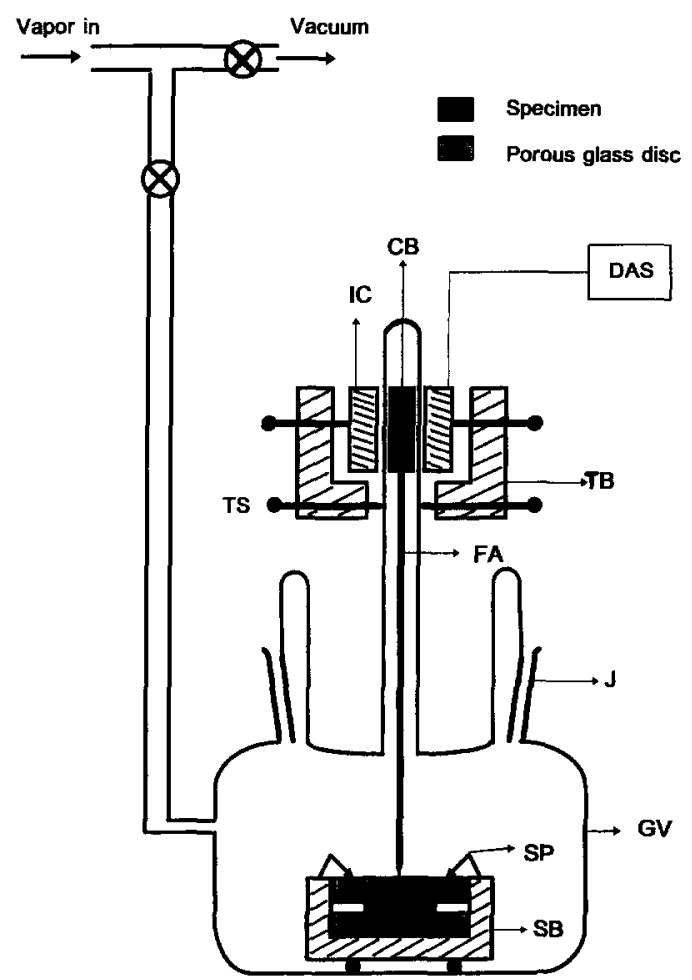

Fig. 2. (a) Schematic representation of the apparatus for gravimetric sorption (A), elongation (B) and thickness dilation (C) measurements (VSA). Q: quartz spring balance; S: film specimen; GV: thermostatted glass vessels; R: thermostatted MC reservoir; AT: air thermostat; TDA: thickness dilation apparatus shown in Fig. 2(b); M: mercury manometer; IT: inductive transducer; and DAS: data aquisition system. (b) Apparatus for thickness dilation measurements. GV: thermostatted glass vessel; SP: spring clips; SB: three-legged base; FA: non-magnetic feeler; IC: transducer induction coil; CB: core of transducer; TB: base for the support of the transducer coil on the glass tube; TS: tightening screws; DAS: data aquisition system; and J: ground glass joint. 
state $\left(n_{\mathrm{P}}, l_{\mathrm{P}}\right)$ and after equilibration with the vapor $\left(n_{\mathrm{M}}, l_{\mathrm{M}}\right)$.

The refractive index $n_{\mathrm{j}}$ of a dielectric substance ' $\mathrm{j}$ ' is related to the molar refraction $R_{\mathrm{j}}$ through the Claussius-Mossotti equation [6,7]

$\frac{n^{2}-1}{n^{2}+2}=\frac{R_{\mathrm{j}}}{M_{\mathrm{wj}}} \rho_{\mathrm{j}}=q_{\mathrm{j}} \rho_{\mathrm{j}}$

where $\rho_{\mathrm{j}}$ is the density; $M_{\mathrm{wj}}$ is the molecular weight; and $q_{\mathrm{j}}$ is a characteristic constant.

The mass concentrations of penetrant and polymer per unit volume of the mixture $c_{\mathrm{c}}$ and $c_{\mathrm{P}}$ respectively are related to the refractive index of the mixture $n_{\mathrm{M}}$, through the following expression, based on additivity of refraction per unit volume upon mixing

$\frac{n_{\mathrm{M}}^{2}-1}{n_{\mathrm{M}}^{2}+2}=c_{\mathrm{c}} q_{\mathrm{c}}+c_{\mathrm{P}} q_{\mathrm{P}}$

where $q_{\mathrm{c}}, q_{\mathrm{P}}$ are the characteristic constants determined from the refractive index $\left(n_{\mathrm{c}}, n_{\mathrm{P}}\right)$ and density $\left(\rho_{\mathrm{c}}, \rho_{\mathrm{P}}\right)$ of the respective pure substances according to Eq. (4). Assuming that the supported polymer film does not dilate significantly in the longitudinal directions

$c_{\mathrm{P}}=\rho_{\mathrm{P}} \frac{l_{\mathrm{P}}}{l_{\mathrm{M}}}$

Eqs. (5) and (6) allow determination of $c_{\mathrm{c}}$ at each activity $a_{i}$ from the corresponding experimental values of $n_{\mathrm{M}}$ and $l_{\mathrm{P}} / l_{\mathrm{M}}$.

\subsubsection{Sorption, swelling and refractive index measurements on free films}

The vacuum sorption/swelling apparatus (VSA) used for the determination of the equilibrium amount of sorbed penetrant and of the corresponding volume swelling is shown schematically in Fig. 2(a).

Gravimetric sorption measurements were performed by means of a quartz spring balance (of capacity ca. $1 \mathrm{~g}$ and sensitivity $25.82 \mathrm{~cm} / \mathrm{g}$ ) read with a cathetometer and yielding a minimum detectable change in weight of ca. $0.04 \mathrm{mg}$.

Swelling measurements were performed on adjoining strips of the same dry CA film. Longitudinal swelling was measured on one of the strips of length $H_{\mathrm{P}} \cong 5.0 \mathrm{~cm}$, by means of an inductive transducer (Schaevitz, type $0300 \mathrm{HR}$, sensitivity $0.282 \mathrm{~V} / \mathrm{mm}$ ).
Changes in the length of the film $\Delta H$ down to $0.01 \mathrm{~mm}$ could be measured (see Ref. [16] for further details). Measurement of swelling in the thickness direction was performed (see Fig. 2(b)) on the other strip (of dry thickness $l_{\mathrm{P}}$ ) placed between two porous glass discs (pressed together with the minimum force necessary to ensure reproducible results), by recording the displacement of a nonmagnetic feeler resting on the upper glass disc and carrying the core of a sensitive inductive transducer (Schaevitz, type 050HR sensitivity $2.2 \mathrm{mV} / \mu \mathrm{m})$. Changes in the thickness of the film $\Delta l$ as low as $0.05 \mu \mathrm{m}$ were detectable. These measurements had to be confined to activities below 0.60 , because of excessive softening of the CA film beyond this range.

The refractive index $n_{M}$ of film samples taken from the same CA film of various degrees of swelling was also measured by immersing film specimens (preequilibrated with various vapor activities in the above apparatus and then clamped between glass plates; see Ref. [9] for further details) in two different liquids (of refractive index $n_{\mathrm{L}}$ and $n_{\mathrm{L}}{ }^{\prime}$ ) and measuring interferometrically (using the Amplival Pol $\mathrm{U}$ microinterferometer of Jena) the optical thickness differences ( $\Delta L_{\mathrm{L}}, \Delta L_{\mathrm{L}}{ }^{\prime}$, respectively) between the film specimen and an equal geometrical thickness of each of the two liquids. Then

$\frac{n_{\mathrm{M}}-n_{\mathrm{L}}}{n_{\mathrm{M}}-n_{\mathrm{L}}^{\prime}}=\frac{\Delta L_{\mathrm{L}}}{\Delta L_{\mathrm{L}}^{\prime}}$

\section{Results and discussion}

Free and supported films of various thicknesses were subjected to series of 'interval' or 'integral' sorption runs, involving successive equilibration with progressively higher vapor activities $a_{i}$, starting with the film in the dry state $(a=0)$. According to the former protocol, equilibration at $a_{i}$ was followed by increase of the vapor activity to $a_{i+1}$; while the latter protocol involved an intervening desorption step back to $a=0$. Neither film thickness $\left(l_{\mathrm{P}}\right)$ nor the experimental protocol were found to affect the results significantly, as illustrated in examples given below.

The sorption isotherm obtained directly from weight gain measurements on the free film, and expressed in terms of the amount of sorbed penetrant 


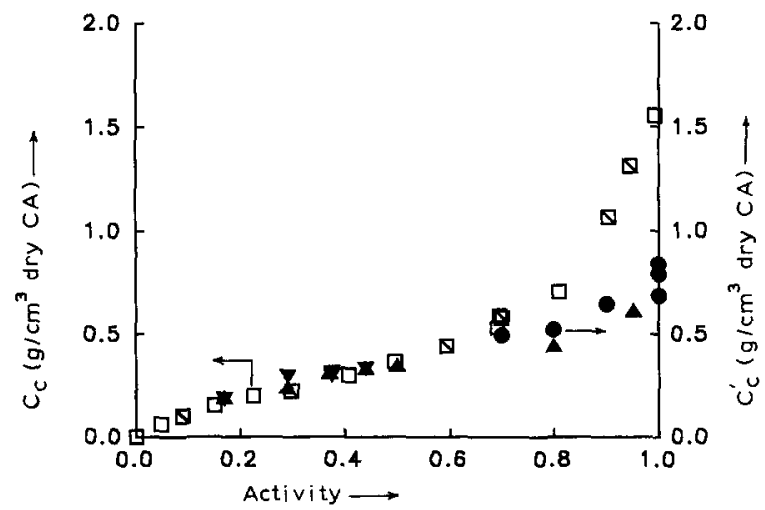

Fig. 3. Examples of equilibrium absorption results at $25^{\circ} \mathrm{C}$ for the system CA/MC obtained by (i) direct gravimetric measurements on free films $\left(l_{\mathrm{P}}=73 \mu \mathrm{m}\right)$ by means of integral runs in the VSA: first series $(\square)$; second series $(\mathbb{\nabla})$ and (ii) application of Eqs. (5) and (6) to data from series of integral $(\boldsymbol{O}, \mathbf{A})$ or interval $(\boldsymbol{\nabla})$ sorption runs on supported film specimens of $l_{\mathrm{P}}=20(\boldsymbol{\nabla}), 23(\mathrm{O})$ or $46(\boldsymbol{\Delta})$ $\mu \mathrm{m}$ in the OTM (see text also).

per unit volume of dry polymer $C_{\mathrm{c}}$ vs. $a$, is illustrated by the open points in Fig. 3. In this particular example, the as-prepared film (of $l_{\mathrm{P}}=73 \mu \mathrm{m}$ ) was subjected to two successive series of integral runs; which agreed well with each other showing that annealing undergone by the film during the first series of runs had no significant effect on the sorption isotherm.

As shown by corresponding elongation and thickness dilation data (obtained from a series of interval sorption runs on a relatively thick $\mathrm{CA}$ film of $l_{\mathrm{P}}=167 \mu \mathrm{m}$, in order to minimize experimentation time and maximize the accuracy of thickness dilation measurement, respectively), the main change brought about by such annealing is a marked decrease in swelling anisotropy between the longitudinal and thickness directions (see Fig. 4). This is due to substantial preferred in-plane macro-molecular orientation of the as-prepared film (see Section 1) which tends to relax under the strong plasticizing action of the penetrant. Note, however, that significant swelling anisotropy persists during the second series of sorption runs; so that estimation of volume swelling on the basis of elongation data alone, designated as assumption (a) in the introductory section, would lead to results significantly in error (up to $10 \%$ ) even for the annealed film.

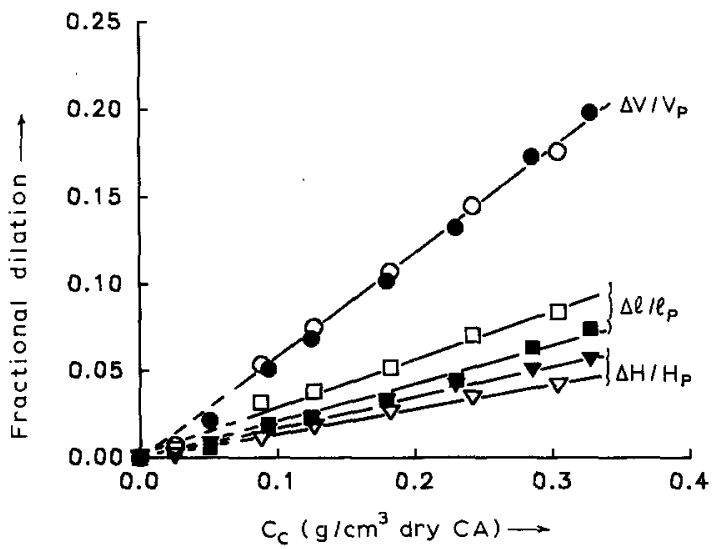

Fig. 4. Concentration dependence of the fractional longitudinal $\Delta H / H_{\mathrm{P}}(\nabla, \nabla)$, thickness $\Delta l / l_{\mathrm{P}}(\square, \square)$ and direct volume swelling $\Delta V / V_{\mathrm{P}}(\mathrm{O}, O)$ of a free film $\left(l_{\mathrm{P}}=167 \mu \mathrm{m}\right)$ obtained from the first series (open points) and the second series (filled points) of interval sorption runs in the VSA. $H_{\mathrm{p}} l_{\mathrm{P}}$ and $V_{\mathrm{p}}$ are the dry film length, thickness and volume, respectively at the beginning of each series.

On the other hand, the fact that (as also shown by Fig. 4) volume swelling properly evaluated is not significantly affected by the swelling anisotropy, explains the agreement between the results of the first and second series of runs in the VSA shown in Fig. 3; and enables us to compare these results with those obtained for supported films by application of Eqs. (5) and (6) to the relevant OTM data. Results of the latter type expressed as $C_{\mathrm{c}}{ }^{\prime}=c_{\mathrm{c}}{ }^{\prime} l_{\mathrm{M}} / l_{\mathrm{P}}$ vs. $a$ (from different film specimens and experimental protocols) are included in Fig. 3. They agree well with the weight gain data up to $a \cong 0.5$ or $C_{\mathrm{c}} \cong 0.4 \mathrm{~g} / \mathrm{cm}^{3}$ dry $C A$. Beyond this point, the OTM sorption isotherm exhibits increasing negative deviation from the VSA one. As indicated in Section 1, this is attributable to failure of assumption (b) i.e. to breakdown Eq. (6); but there is also possibility (c), namely, that the supported film cannot attain full swelling equilibrium, as a result of the constraint imposed on longitudinal swelling.

The latter possibility was checked by measuring the refractive index of free films, equilibrated with different vapor activities in the VSA, by the two-liquid method described in the Section 2. The results obtained (using dibutyl phthalate and sec-butanol as the two liquids) are shown in Fig. 5(a) together with those derived from the OTM via Eqs. (2) and (3), as a function of the respective weight fractions $\omega_{c}$ and 

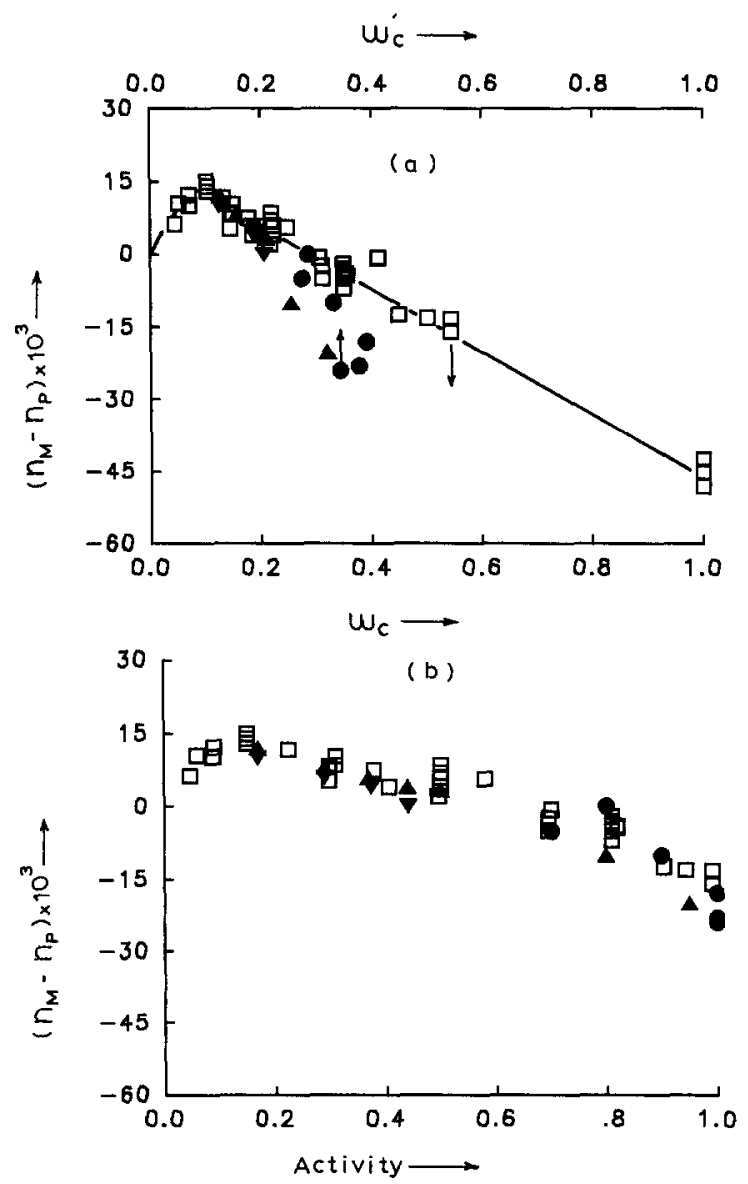

Fig. 5. (a) Comparison of refractive index increments $\left(n_{\mathrm{M}}-n_{\mathrm{P}}\right)$ measured (i) on free film (open points), or (ii) on supported film (filled points), equilibrated with different $\mathrm{MC}$ vapor activities in the VSA or OTM respectively, plotted as a function of MC mass fraction (i) measured directly in the VSA $\left(\omega_{c}\right)$ or (ii) deduced from OTM data $\left(\omega_{c}^{\prime}\right)$. (b) The refractive index data of Fig. 5(a) replotted as a function of vapor activity. Symbols and experimental protocol as in Fig. 3.

$\omega_{\mathrm{c}}{ }^{\prime}=1 /\left(1+\rho_{\mathrm{P}} / C_{\mathrm{c}}{ }^{\prime}\right)$ (derived from the VSA and OTM measurements, respectively). As might be expected, the two sets of results agree at low concentrations up to the point where the VSA and OTM sorption isotherms in Fig. 3 diverge. Beyond this point the OTM refractive index plot deviates negatively from the VSA one. The latter plot is the correct one, as is confirmed by the fact that, as shown in Fig. 5(a), it extrapolates smoothly through the value for pure liquid penetrant $n_{\mathrm{c}}$. Hence, it appears that the negative deviations of the OTM from the VSA data in Figs. 3 and 5(a) are largely attributable to the fact that the $C^{\prime}$ values deduced from the OTM data in this region are underestimates of the corresponding actual $C$ values, due to breakdown of Eq. (6). The fact that equilibrium volume swelling of the supported film is not less than that of the free film is shown most clearly by comparison of the respective refractive index values as a function of vapor activity (see Fig. 5(b)).

In keeping with the above conclusion, the degree of volume swelling inferred from the OTM data on the basis of Eq. (6), when plotted as a function of the actual concentration $C_{\mathrm{c}}$ (derived from the VSA), agrees with the corresponding results of volume swelling measured by the VSA at lower concentrations, but deviates markedly from the extrapolated VSA plot in the higher concentration region (see Fig. 6). As explained in Section 2, swelling measurements by the VSA were not reliable in the latter region because of excessive softening of the film; Fig. 6 shows that estimates of volume swelling of the supported film derived from the OTM also become unreliable in approximately the same concentration region.

The results pertaining to the concentration region where agreement between swelling data from the VSA and corresponding estimates from the OTM was satisfactory, are replotted in Fig. 7 as a function of

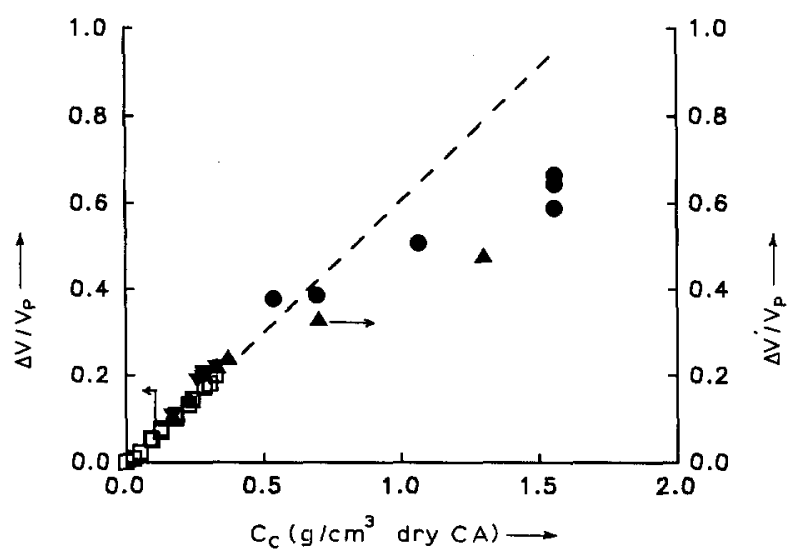

Fig. 6. Comparison of fractional volume swelling (i) measured directly on free film in the VSA $\left(\Delta V / V_{\mathrm{P}}\right.$, open points) or (ii) inferred from thickness dilation measurements on supported film in the OTM ( $\Delta V^{\prime} / V_{\mathrm{P}}=\Delta l / l_{\mathrm{P}}$; full points), as a function of $\mathrm{MC}$ concentration $\left(C_{\mathrm{c}}\right)$ measured directly in the VSA. Symbols and experimental protocol as in Fig. 3. 


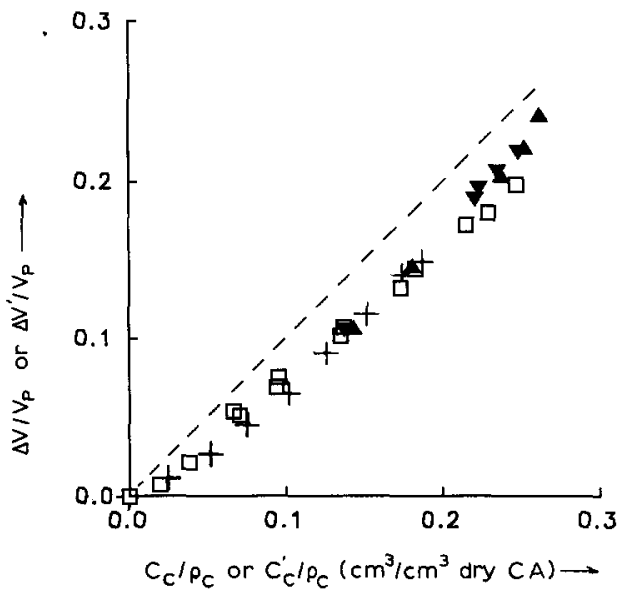

Fig. 7. Fractional volume dilation of CA swollen by $\mathrm{MC}(\square, \nabla$, $\boldsymbol{\Delta}$; present work) or ethanol (+) [7], measured directly on free film in the VSA ( $\square$ ) or deduced from optical measurements on supported films $(\boldsymbol{\nabla}, \boldsymbol{\Delta},+)$, plotted as a function of volume of liquid penetrant taken up by unit volume of dry polymer. Experimental details concerning the MC data as in Fig. 3. The ethanol data pertain to CA films $\left(l_{\mathrm{P}}=2-4 \mu \mathrm{m}\right)$ spun under $\mathrm{N}_{2}$ from dimethyl formamide dope on a quartz plate, then dried and annealed by heat treatment at $180^{\circ} \mathrm{C}$ for $30 \mathrm{~min}$. The result expected if additivity of volumes is preserved in the mixing process is indicated by a broken line of unit slope.

$C / \rho_{\mathrm{c}}$ and $C^{\prime} / \rho_{\mathrm{c}}$, respectively, which represent the fractional volume swelling expected if the condition of additivity of volume holds for the polymer-penetrant mixing process and permits comparison of the deviations from this condition observed with different liquid penetrants. In Fig. 7, the present results are compared with those reported by Bolton et al. for CA of the same composition penetrated by ethanol [7]. It will be noted that the latter data were obtained optically on supported films and cover degrees of swelling well within the range where the present OTM results based on assumption (b) were found to be reliable. The two sets of results agree reasonably well, indicating that filling of the excess free volume of the polymer proceeds similarly in these two cases.

\section{Conclusion}

The work reported here involves use of optical thickness meter (OTM) for the combined measure- ment of the thickness and refractive index of supported CA films equilibrated with various activities of a strong swelling agent, namely $\mathrm{MC}$. The relevant volume swelling and sorption isotherms could be derived from the results obtained, on the assumption that these films swell unidimensionally along the thickness direction (Eq. (6)) followed by application of the Claussius-Mossotti relation (Eq. (5)).

Parallel direct measurements of the aforementioned isotherms were also performed on free CA films in a vacuum sorption/swelling apparatus (VSA) and complemented with refractive index data. Combined elongation and thickness dilation data from the VSA showed that free glass-cast CA films exhibit pronounced swelling anisotropy, which (i) can be markedly reduced, but not completely eliminated, by conditioning at high degrees of swelling, and (ii) does not appear to affect the extent of volume swelling significantly. These findings lead to the corresponding conclusions that (i) the assumption often made in practice that films annealed by heat or conditioning treatments may be considered to be isotropic, thereby enabling one to determine volume swelling from elongation measurements alone, require careful checking in each particular case (e.g. by measuring the birefringence of a cross section of the film) and (ii) quantitative comparison of sorption and swelling isotherms determined by the VSA and the OTM is permissible. Such comparison showed satisfactory agreement between these two sets of results up to an MC uptake of ca. $0.4 \mathrm{gMC} / \mathrm{cm}^{3}$ of dry CA corresponding to a degree of swelling of ca. 0.2. Increasing discrepancies are observed at higher MC concentrations, which are attributable to breakdown of Eq. (6) (rather than to limited volume swelling of the supported film resulting from the constraint imposed on longitudinal swelling). It follows that the determination of equilibrium sorption and volume isotherms by means of the OTM can be relied upon only at low to moderate degrees of swelling.

The present CA-MC volume swelling data exhibit the negative deviation from volume additivity upon mixing typical of glassy polymers and closely resemble the corresponding data of Bolton et al. [7] for CAethanol (derived from optical data on supported films at degrees of swelling well within the reliability limits indicated above). 


\section{Acknowledgements}

E.U. support for this work, including a postdoctoral fellowship for D. Stamatialis, within the framework of the Human Capital and Mobility Project entitled 'Functional Membranes,' is gratefully acknowledged.

\section{References}

[1] W.R. Vieth, Dual mode sorption theory, J. Membrane Sci., 1 (1976) 177.

[2] D.P. Paul, Gas sorption and transport in glassy polymers, Ber. Bunsenges. Phys. Chem., 83 (1976) 294.

[3] G.K. Fleming and W.J. Koros, Dilation of polymers by sorption of carbon dioxide at elevated pressures. 1. Silicone rubber and unconditioned polycarbonate, Macromolecules, 19 (1986) 2285.

[4] G.K. Fleming and W.J. Koros, Dilation of substituted polycarbonates caused by high-pressure carbon dioxide sorption, J. Polym. Sci., Polym. Phys. Ed., 28 (1990) 1137.

[5] Y. Kamiya, T. Hirose, Y. Naito and K. Mizoguchi, Sorptive dilation of polysulfone and poly(ethylene terephthalate) films by high-pressure carbon dioxide, J. Polym. Sci., Polym. Phys. Ed., 26 (1988) 159.

[6] J.R. Scherer and G.F. Bailey, Water in polymer membranes. Part I. Water sorption and refractive index of cellulose acetate, J. Membrane Sci., 13 (1982) 29.

[7] B.A. Bolton, S. Kint, G.F. Bailey and J.R. Scherer, Ethanol sorption and partial molar volume in cellulose acetate films, J. Phys. Chem., 90 (1986) 1207.

[8] C. Robinson, Interferometric studies in diffusion. I. Determination of concentration distributions, Proc. Roy. Soc. London, A204 (1950) 339.
[9] D.F. Stamatialis, M. Sanopoulou and J.H. Petropoulos, Phenomena affecting the interferometric determination of concentration profiles of micromolecules diffusing along a stiff-chain polymer film, J. Appl. Polym. Sci., in press.

[10] D.S. Pope, W.J. Koros and G.K. Fleming, Measurement of thickness dilation in polymer films, J. Polym. Sci., Polym. Phys. Ed., 27 (1989) 1173.

[11] R.G. Wissinger and M.E. Paulaitis, Swelling and sorption in polymer- $-\mathrm{CO}_{2}$ mixtures at elevated pressures, J. Polym. Sci., Polym. Phys. Ed., 25 (1987) 2497.

[12] Y. Kamiya, K. Mizoguchi, T. Hirose and Y. Naito, Sorption and dilation in poly(ethylmethacrylate)-carbon dioxide system, J. Polym. Sci., Polym. Phys. Ed., 27 (1989) 879.

[13] L.C. Witchey-Lakshmanan, H.B. Hopfenberg and R.T. Chern, Dilation of poly[1-(trimethylsilyl)-1-propyne] during sorption of $n$-nonane, J. Polym. Sci., Polym. Phys. Ed., 31 (1993) 1545.

[14] M. Sanopoulou and J.H. Petropoulos, Sorption and longitudinal swelling kinetic behavior in the system cellulose acetate-methanol, Polymer, in press.

[15] F.A. Long and R.J. Kokes, Diffusion of methylene chloride vapor into polystyrene, J. Am. Chem. Soc., 75 (1953) 2232.

[16] M. Sanopoulou, P.P. Roussis and J.H. Petropoulos, A detailed study of the viscoelastic nature or vapor sorption and transport in a cellulosic polymer. II. Sorption and longitudinal swelling kinetic correlations, J. Polym. Sci., Polym. Phys. Ed., 33 (1995) 2125.

[17] R.M. Ikeda and F.P. Gay, Vapor permeation in polymer films, J. Appl. Polym. Sci., 17 (1973) 3821.

[18] M.D. Sefcik, Dilation of polycarbonate by carbon dioxide, J. Polym. Sci., Polym. Phys. Ed., 24 (1986) 935.

[19] M. Born and E. Wolf, Principles of Optics, Pergamon, New York, 1970. 